\title{
Echoes of the Brain within Default Mode, Association, and Heteromodal Cortices
}

\author{
Rodrigo M. Braga, ${ }^{1,2}$ David J. Sharp, ${ }^{1}$ Clare Leeson, ${ }^{1}$ Richard J.S. Wise, ${ }^{1}$ and Robert Leech ${ }^{1}$ \\ ${ }^{1}$ Computational, Cognitive and Clinical Neuroimaging Laboratory, and ${ }^{2}$ Medical Research Council Clinical Sciences Centre, Faculty of Medicine, Imperial \\ College London, Hammersmith Hospital Campus, London, W12 0NN, United Kingdom
}

Intrinsic connectivity networks (ICNs), such as the default mode, frontoparietal control, and salience networks, provide a useful largescale description of the functional architecture of the brain. Although ICNs are functionally specialized, the information that they process needs to be integrated for coherent cognition, perception, and behavior. A region capable of performing this integration might be expected to contain traces, or "echoes," of the neural signals from multiple ICNs. Here, using fMRI in humans, we show the existence of specific "transmodal" regions containing echoes of multiple ICNs. These regions include core nodes of the default mode network, as well as multimodal association regions of the temporoparietal and temporo-occipito-parietal junction, right middle frontal gyrus, and dorsal anterior cingulate cortex. In contrast, "unimodal" regions such as the primary sensory and motor cortices show a much more singular pattern of activity, containing traces of few or even single ICNs. The presence of ICN echoes might explain how transmodal regions are involved in multiple different cognitive states. Our results suggest that these transmodal regions have a particular local spatial organization containing topographic maps that relate to multiple ICNs. This makes transmodal regions uniquely placed to be able to mediate the cross talk between the brain's functional networks through local modulation of adjacent regions that communicate with different ICNs.

\section{Introduction}

Human cognition and behavior involves the coordination of activity across large-scale brain networks (Mesulam, 2009; Bressler and Menon, 2010). These networks can be identified using fMRI (Damoiseaux and Greicius, 2009) and have become known as intrinsic connectivity networks (ICNs). Some of the typical ICNs, such as the frontoparietal attention, language, salience, motor, primary sensory, and default mode networks, are observable during both the presence and absence of a cognitive task (Raichle et al., 2001; Fransson, 2005; Smith et al., 2009, 2012). This has led to the theory that ICNs make up the brain's macroscopic functional architecture, with subsets of ICNs recruited based on task demands (Spreng et al., 2010; Leech et al., 2012; de Pasquale et al., 2012).

Although these ICNs may be functionally specialized, the information that they process needs to be integrated for coherent cognition, perception, and behavior. A region capable of performing this integration might be expected to contain traces, or "echoes," of the neural signals from multiple ICNs. If so, then these multiple signals should be observable within a given cortical

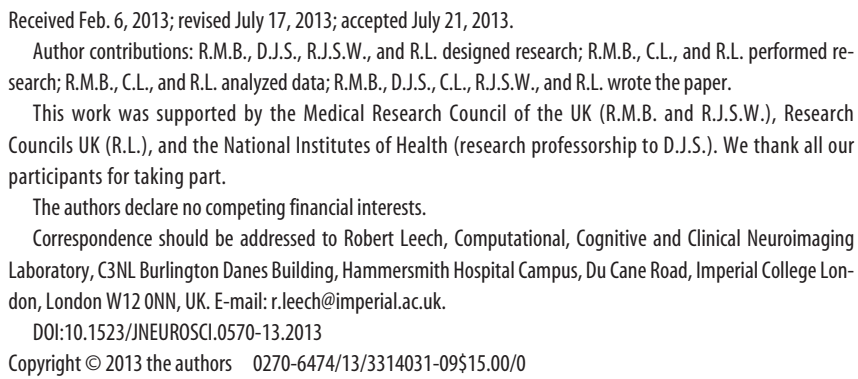

region. These regions will therefore be candidate "connector hubs," in the terminology proposed by Sporns et al. (2007), bridging different ICN "modules" in a focal cortical center.

We have previously shown that multiple ICN echoes can be observed within a relatively small region of parietal paralimbic cortex, the posterior cingulate cortex (PCC; Leech et al., 2012). The existence of spatially neighboring subregions of the PCC that communicate with whole-brain ICNs suggests that the PCC may be mediating the cross talk between many different functional networks (Margulies et al., 2009; Leech et al., 2011). Although traces of multiple ICNs can be found within the PCC, the PCC has the highest metabolism in the cortex (Raichle et al., 2001) and some of the densest structural connections (Vogt et al., 2006; Hagmann et al., 2008) and it is unknown which other brain regions, if any, share this property.

In the present study, we investigated which regions of cortex contain multiple signals that relate to the ICNs. Our hypothesis is that this property will not be ubiquitous, but will be most pronounced within regions previously thought to be "heteromodal" or "transmodal" (Mesulam, 1998). These may include the multimodal association areas, the temporo-occipito-parietal junction (TOPJ), and structural "cores" in the anterior and posterior cingulate cortices (Hagmann et al., 2008; Sepulcre et al., 2012). In contrast, unimodal regions such as the primary sensory cortices will display a much more singular pattern of whole-brain functional connectivity, with few ICN echoes observable within these regions.

\section{Materials and Methods}

Overview

Figure 1 gives an overview of the experiment. Twenty-five subjects were scanned using fMRI while at rest. Independent component analysis 

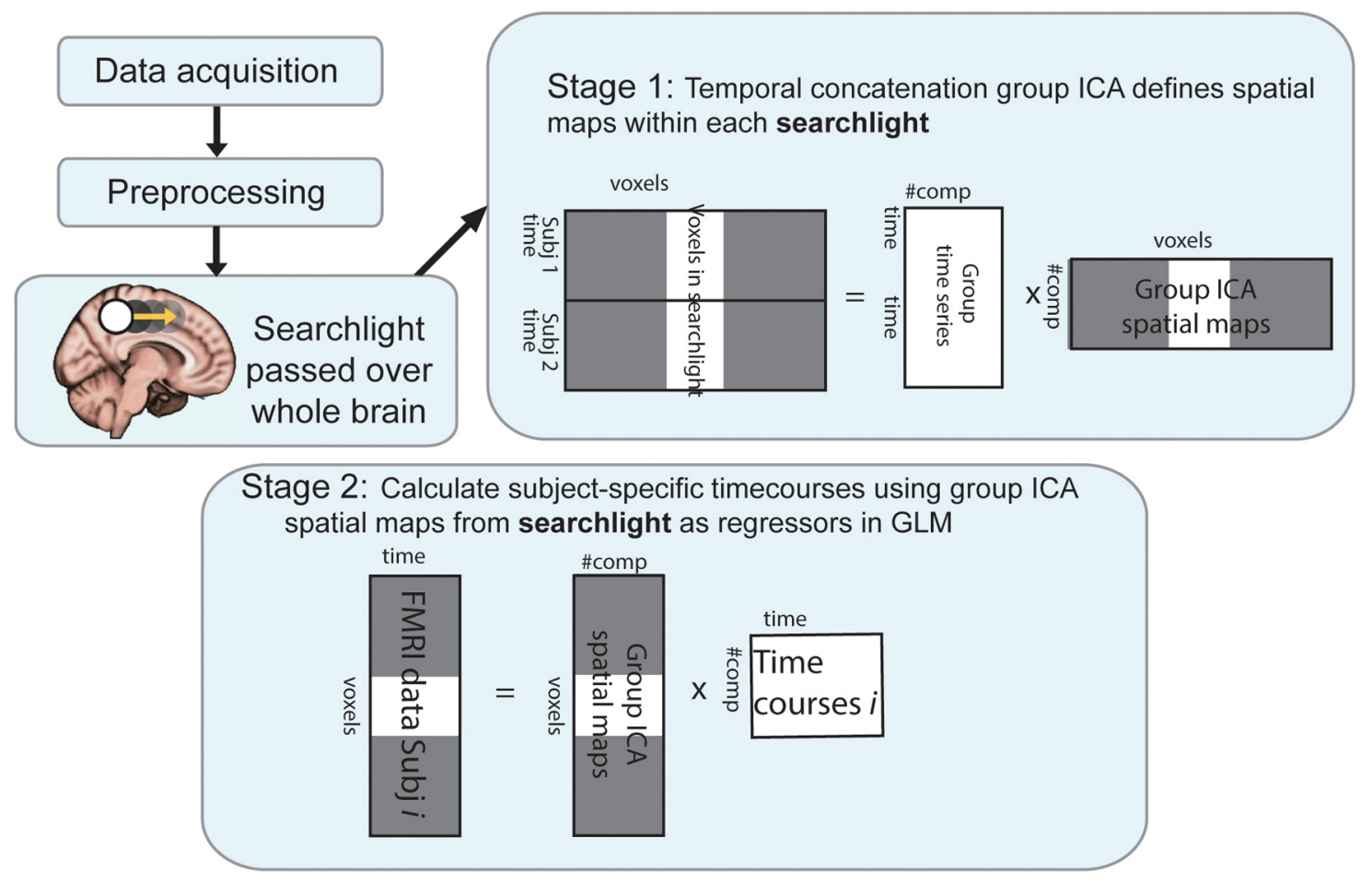

Stage 3: Calculate subject-specific whole-brain spatial maps using subject-specific time courses as regressors in GLM

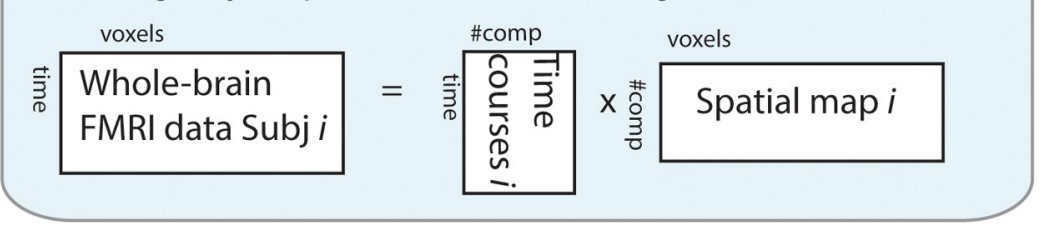

\section{Stage 4: Higher-level GLM across subjects, using subject specific whole-brain spatial maps.}

Figure 1. Schematic of FC analysis steps. A group temporal concatenation ICA was performed to find separable spatial components within each spherical searchlight. These components were then back projected to find a subject-specific time course for each searchlight component. Time courses were then simultaneously entered into a GLM voxelwise throughout the brain, resulting in a FC map for each component.

(ICA) was used to decompose the BOLD signal from a 16-mm-diameter searchlight into 10 components (Smith et al., 2004; Beckmann et al., 2005; Leech et al., 2012). A dual regression technique (Beckmann et al., 2009) was used to assess the functional connectivity (FC) of these 10 components from within the searchlight with the whole-brain dataset (Fig. 1 and see below). The resulting FC maps were then labeled using spatial correlation with well recognized ICNs taken from whole-brain ICAs (WBICAs) from our resting dataset, whole-brain resting-state ICAs from Smith et al. (2009), and aggregated task-based data from BrainMap (also from Smith et al., 2009). This searchlight was then passed around the whole brain in 5 voxel steps. We repeated this procedure using fMRI data obtained during a choice reaction time (CRT) task (a forced choice visuomotor decision task that reliably activates visual, motor, and many cognitive control regions). This allowed us to investigate whether the subregions within each searchlight were functionally meaningful (i.e., whether the local topographic organization and whole-brain FC maps were consistent during and/or modulated by a cognitive task).

\section{Subjects}

A group of 25 neurologically healthy subjects took part in the resting state study (12 males, age range 19-49 years, mean age 32.7 years). A group of 15 neurologically healthy subjects took part in the CRT study (7 males, age range $27-58$ years, mean age 35.0 years). Both studies were approved by the Hammersmith and Queen Charlotte's and Chelsea Research ethics committees.

\section{MRI data acquisition}

MRI data were obtained using a Philips Intera 3.0 tesla MRI scanner using Nova Dual gradients, a phased array head coil, and sensitivity encoding (SENSE) with an undersampling factor of 2. Functional MRI images were obtained using a $\mathrm{T} 2{ }^{*}$-weighted gradient-echo echoplanar imaging (EPI) sequence with whole-brain coverage (TR/TE $=2000 / 30 ; 31$ ascending slices with thickness $3.25 \mathrm{~mm}$, gap $0.75 \mathrm{~mm}$, voxel size $2.19 \times 2.19 \times 4 \mathrm{~mm}$, flip angle $90^{\circ}$, field of view $280 \times 220 \times 123 \mathrm{~mm}$, matrix $112 \times 87$ ). Quadratic shim gradients were used to correct for magnetic field inhomogeneities within the brain. T1-weighted whole-brain structural images were also obtained in all subjects. The CRT paradigm was programmed using the MATLAB (MathWorks) Psychophysics toolbox (Psychtoolbox-3; www. psychtoolbox.org) and stimuli were presented through an IFIS-SA system (in vivo). Responses were recorded through a fiber optic response box (NordicNeuroLab) interfaced with the stimulus presentation PC running MATLAB.

In the resting state scan, 300 volumes were acquired while subjects lay in the scanner with their eyes closed. In the CRT task, acquired during a 
different scanning session, a subset of the subjects were presented with an initial fixation cross for $350 \mathrm{~ms}$. This was followed by a response cue in the form of an arrow $(<<<$ or $>>>)$ in the direction of the required response and lasting $1400 \mathrm{~ms}$. The interstimulus interval was $1750 \mathrm{~ms}$. Finger-press responses were made with the index finger of each hand. Subjects were instructed to respond as quickly and as accurately as possible. To maximize design efficiency, stimulus presentation was blocked, with five repeated blocks of 14 response trials, 14 rest trials, and four response trials at the start of the experiment, resulting in 74 response trials in total.

\section{FC analysis (Fig. 1)}

Data preprocessing. Image preprocessing involved realignment of EPI images to remove the effects of motion between scans, spatial smoothing using a $6 \mathrm{~mm}$ full-width half-maximum Gaussian kernel, prewhitening using FMRIB's Improved Linear Model, and temporal high-pass filtering using a cutoff frequency of $1 / 50 \mathrm{~Hz}$ to correct for baseline drifts in the signal. FMRIB's Linear Image Registration Tool (Smith et al., 2004) was used to register EPI functional datasets into standard MNI space using the participant's individual high-resolution anatomical images.

In addition, variance associated with motion (six variables) and the time courses of white matter and CSF were removed from the wholebrain functional data using ordinary least-squares linear regression. Mean time courses were extracted from a 3-mm-radius sphere within the white matter (MNI $-26,-22,28)$ and from one lateral ventricle (MNI 2, $10,8)$. Average whole-brain or gray matter activity was not regressed because this can complicate the interpretation of negative functional correlation findings (Murphy et al., 2009).

Finding subregions within each searchlight. A grid of 16-mm-diameter (251 voxel) spherical searchlights was created and spaced 5 voxels apart covering the whole brain. These spheres were masked so that voxels outside the brain were not considered. A temporal concatenation group ICA was then run on the resting fMRI data within each searchlight using FMRIB's Melodic software (Beckmann et al., 2005; version 3.1, using default settings and variance normalization). This approach identified group-derived componentswhich we call subregions below-within each sphere based on their temporal coactivation (Fig. 1, Stage 1). The analysis was constrained to extract 10 independent components based on previous work that investigated the effect of using different levels of dimensionality (Leech et al., 2012). Fixing the number of independent components extracted allowed us to investigate regional variation in the presence of whole-brain signals for different searchlights (i.e., which regions have similar properties of whole-brain echoes as our previous findings about the PCC; Leech et al., 2012). Therefore, our approach was not designed to define the precise number of whole-brain maps (which could be $>10$ in some cases). However, qualitatively similar results were also found when the analysis was repeated using different sized spherical searchlights of 500 voxels.

Finding whole-brain FC for searchlight subregions. For the next step, a general linear model (GLM) was used with the fMRI data, simultaneously including the 10 independent component spatial maps from within a specific searchlight as a design matrix; this approach results in a subjectspecific time course for each independent component (Fig. 1, Stage 2). We then investigated which voxels from across the whole brain were functionally connected to these subject-specific time courses. To do this, a second GLM stage used the time courses for each voxel from the wholebrain fMRI data (not just the searchlight) as a dependent variable. The subject-specific time courses of the searchlight components calculated in the previous step were simultaneously included in the design matrix, resulting in a set of whole-brain subject-specific spatial maps (Fig. 1, Stage 3). This approach, also used in Leech et al. (2012), is a variant on the dual regression approach (Zuo et al., 2010) used by Leech et al. (2011) and Bonnelle et al. (2011). In the final stage, the subject-specific spatial maps were entered into a higher-level GLM (Beckmann et al., 2003). This voxelwise higher-level model assessed whether the average FC values across subjects differed significantly from zero (Fig. 1, Stage 4). This resulted in a group whole-brain FC statistical parametrical map for the different independent components from within the searchlight.

Summary statistics and whole-brain maps. To interpret the findings, we labeled the resulting group-averaged whole-brain FC maps in a princi- pled way with reference to existing ICNs, defining the ICNs in three ways as follows. First, the set of whole-brain FC spatial maps corresponding to each searchlight was correlated with the results from running a group temporal concatenation ICA on the whole-brain data with 20 components (WBICAs). Second, the consistency of this labeling was also assessed by correlating the whole-brain FC maps with the ICNs identified in Smith et al. (2009) from fMRI resting state networks (RSNs) using WBICA . The labeling of the whole-brain ICNs identified in Smith et al. (2009) (e.g., identifying the right frontoparietal network as related to attentional control) has been validated with regard to a large database of task data from the BrainMap database, which was also used to label the whole-brain FC maps in the present study. Following the standards described in Smith et al. (2009), any ICN maps that included a majority of white matter or CSF voxels or were located mostly outside of the brain were deemed to be artifactual and were excluded from further analysis. This left seven ICN maps from the WBICA, 12 from the RSNs, and 13 from the BM datasets.

To summarize how much the whole-brain FC maps from each sphere resembled (or echoed) the whole-brain ICNs, we calculated the maximum spatial correlation between each target ICN and the 10 FC maps. To generate a simple summary statistic, we then calculated the median of these maximum spatial correlations between the ICNs and each of the 10 FC maps for each searchlight. A high median value therefore reflects searchlights for which many FC maps had a high spatial correlation with one or more ICNs. In addition to presenting the median value, we also used an arbitrary spatial correlation threshold and counted the number of FC maps that spatially correlated with the ICNs above this value. We present the statistical maps of these counted values at an arbitrary threshold of $r>0.3$. We also calculated this median of maximum spatial correlations with the RSNs and grouped task-based data from the BrainMap database (taken from Smith et al., 2009). Given that the exact value of the spatial correlation is relatively uninterpretable (with the precise value being dependent on how the target ICN data were acquired), different thresholds were used to best show the distribution of the peak regions for the three target ICNs. However, the unthresholded maps for each ICN target maps are also included.

The primary aim of the analyses was to identify which regions, if any, contain multiple whole-brain FC maps that resemble the ICNs, as we found previously for the PCC. We also sought to quantify the general distribution of which searchlight regions contain evidence of multiple whole-brain FCs. However, we also evaluated which specific searchlights contained evidence for a greater number of whole-brain FCs. We used a random permutation approach to test the null hypothesis that there was no difference in the median of the maximum spatial correlation across different searchlights. To do this, the maximum correlation values were Fisher transformed, shuffled across searchlights 20,000 times, and the median correlation for each searchlight for each permutation was calculated. This built up a null distribution of median maximum values against which the true median maximum spatial correlations with the target ICNs could be evaluated. The resulting statistical maps were thresholded either at a liberal threshold of $p<0.01$ (uncorrected for multiple comparisons) and corrected for multiple comparisons using a false discovery rate with $q=0.1$ and $q=0.05$.

\section{Task modulation of subregion activation}

The CRT data were preprocessed in exactly the same way as the resting state data. The whole-brain pattern of relative activation evoked by the task has been presented previously (Leech et al., 2012). The same searchlight-constrained dual regression analysis (Fig. 1) was repeated on this task dataset, yielding subject-specific time courses and subjectspecific whole-brain FC maps from within each searchlight subregion. Within the nine representative peak searchlights identified from the resting state data, time series were assessed for modulation during the CRT task using the GLM (Leech et al., 2012). The subject-specific time course for each component (Fig. 1, Stage 2) was the dependent variable, and the blocked time course of the CRT task and its first temporal derivative were included in the design matrix. Each subject's parameter estimate for the CRT time course was then used in a higher-level GLM across subjects, assessing whether that component was significantly modulated by the 
CRT task. The $p$-values resulting from this higher-level analysis were Bonferroni corrected for multiple comparisons (for the 10 independent components) and presented at $p<0.05$.

Our approach decomposes the activity within a searchlight in a data-driven way into spatial subregions and associated time courses that flexibly allows for a range of questions to be addressed. For example, these subregions and time courses can be used to assess wholebrain FC (e.g., to determine whether the subregions are connected to multiple ICNs). However, in addition, they allowed us to assess how the searchlight is spatially organized in terms of the locations of the subregions, how the time courses are affected by different tasks, and potentially (although we do not consider this here) how the time series from different subregions interact and how these interactions change with task. An alternative, simpler approach to identifying "hub" regions communicating with multiple ICNs would be to use whole-brain spatial ICA to identify neural components, threshold and binarize the components, and then count how many components are present within each searchlight. Although this alternative approach could potentially address the specific question of which regions contain multiple whole-brain ICNs, the present approach allows additional questions to be answered (as described above). In addition, the subregions delineated by our approach are not determined by activity and noise in distal brain regions. This means that the resulting whole-brain FC maps from each searchlight can contain a mixture of frequently occurring ICNs, nonstandard wholebrain maps, and local maps (Simmonds et al., 2013). In addition, the present approach produces subregion components that control for the variance in the other subregions. Therefore, the time series obtained from each subregion are not dominated by the principal pattern of activation within that cortical region, meaning that more subtle subregional effects (e.g., connectivity with different whole-brain ICNs) can be detected. These subtle interactions are not observable if these local different sources of variability are not controlled for (Leech et al., 2012).

\section{Results}

\section{Considerable variations in which regions contain multiple ICN signals}

Figure 2 presents the regional distribution of the extent to which regions contain multiple whole-brain FC similar to ICNs. This distribution is presented both for the median maximum spatial correlations (Fig. 2, top) and by counting the number of ICNs present in each searchlight over an arbitrary threshold of $r>0.3$ (Fig. 2, middle). The resulting images illustrate which areas contain multiple whole-brain FC maps resembling ICNs (hot areas) and those that show relatively few ICNs (cold areas). Figure 2, bottom, presents searchlights that display significantly elevated median correlations at various thresholds. Table 1 shows the coordinates of the significant spheres from Figure 2, bottom.

Illustrative peak regions from Figure 2 with high median correlations were explored in subsequent analyses. Regions with evidence of greater numbers of ICNs included core nodes of the DMN (the PCC and precuneus) multimodal association regions around the TOPJ, right middle frontal gyrus (MFG), and dorsal anterior cingulate cortex (ACC). In contrast, regions with evidence of fewer ICNs included bilateral primary visual centers, right primary auditory cortex, motor and somatosensory corti-
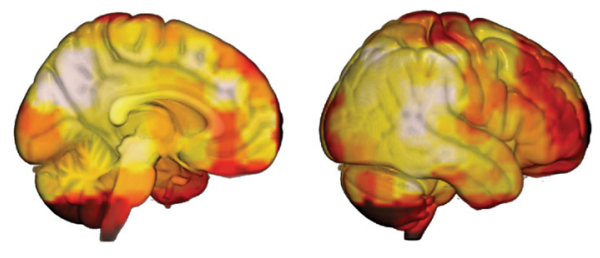

0.4
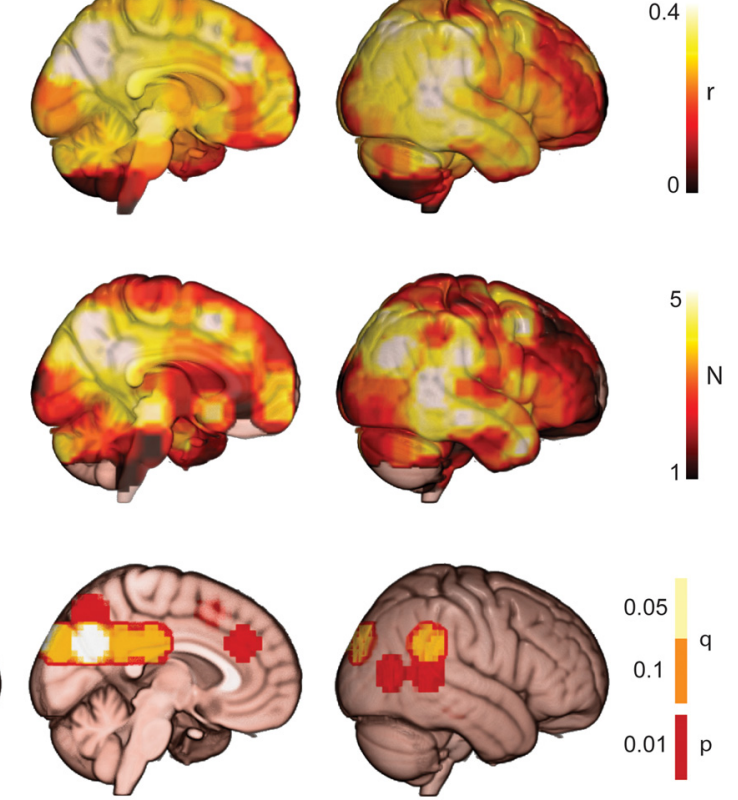

\section{(1)}

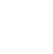

Figure 2. Surface rendering showing peak regions where the highest amount of overlap of signals from the canonical ICNs were the median (top) and number of correlations above an arbitrary threshold of $r>0.3$ (middle) were plotted on each searchlight. clevated median spatial correlations with ICNs (bottom). These were thresholded at $p<0.01$, uncorrected, and using false discovery rate correction with $q=0.1$ and $q=0.05$.

Table 1. Table showing coordinates of spheres containing significantly elevated median spatial correlations with ICNs as illustrated in Figure 2, bottom

\begin{tabular}{llrrrl}
\hline Name & Hemisphere & $x$ & \multicolumn{1}{l}{$y$} & $z$ & Confidence \\
\hline PCC & $\mathrm{R}$ & 10 & -66 & 28 & $* * *$ \\
TPJ & $\mathrm{L}$ & -30 & -86 & 28 & $* * *$ \\
Precun & $\mathrm{L}$ & -10 & -86 & 28 & $* * *$ \\
TOPJ & $\mathrm{L}$ & -50 & -66 & 8 & $* * *$ \\
pMTG & $\mathrm{R}$ & 50 & -26 & -12 & $* *$ \\
Supp Latt 0cc & $\mathrm{R}$ & 30 & -86 & 28 & $* *$ \\
Precun & $\mathrm{R}$ & 10 & -86 & 28 & $* *$ \\
TPJ & $\mathrm{R}$ & 70 & -46 & 28 & $* *$ \\
SPL & $\mathrm{L}$ & -30 & -46 & -48 & $*$ \\
pSTG & $\mathrm{L}$ & -70 & -26 & 8 & $*$ \\
ACC & $\mathrm{L}$ & -10 & 14 & 48 & $*$ \\
TOPJ & $\mathrm{R}$ & 50 & -66 & 8 & $*$ \\
ACC & $\mathrm{R}$ & 10 & 34 & 28 & $*$ \\
PreC & $\mathrm{L}$ & -50 & -6 & 28 & $*$ \\
\hline
\end{tabular}

Where multiple spheres overlapped in the same cortical area, only the highest level of confidence is displayed ${ }^{*} p<0.01$ (uncorrected), corrected using false discovery rate at ${ }^{* *} q=0.1$ and ${ }^{* * *} q=0.05$.

Precun, precuneus; pMTG, posterior MTG; Supp Latt Occ, superior lateral occipital cortex; SPL, superior parietal lobe; pSTG, posterior superior temporal gyrus; PreC, precentral gyrus.

ces, and much of the anterior frontal lobe. As an illustration, Figure 3 shows example whole-brain FC maps from regions where high spatial correlation was observed with ICNs (i.e., five of the peaks from Fig. 2). In these regions, many of the 10 wholebrain FC maps resembled whole-brain ICNs. Qualitatively similar whole-brain FC maps were also found when a 500 voxel spherical searchlight was used instead.

\section{Echoes of different ICNs detected in different heteromodal regions}

Figure 4 shows the similarity matrix between 12 resting state ICNs (taken from Smith et al., 2009) and the whole-brain FC 


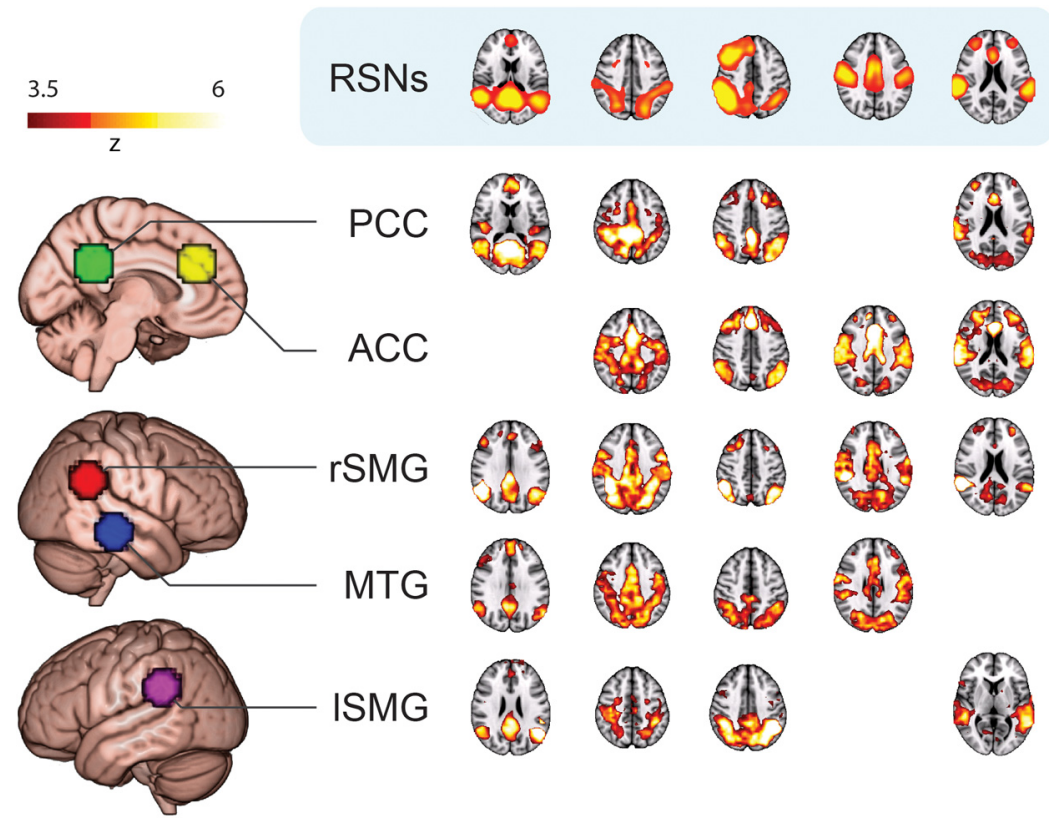

Figure 3. Example $F($ maps obtained from five of the nine peak regions observed in Figure 2. Many of these $F($ maps resembled the whole-brain ICNs detected from a WBICA of the same data. Peak regions shown are the PCC and ACC, the right SMG (rSMG), left SMG (ISMG), and right MTG. RSNs are (left to right) the default mode, visual streams, right frontoparietal, motor and auditory/ salience networks, which are displayed for illustrative purposes (labeled by spatial correlation to BrainMap data in Smith et al., 2009). Colorbar shows $z$-scores. FC maps are thresholded at $z>3.5$. Correlation values for each peak region for each ICN map are displayed in Figure 4.

maps observed in the nine illustrative regions from Figure 2. Each peak region contained signals from different combinations of ICNs, indicating that each peak hub mediates cross talk between different functional groups. The PCC, ACC, supramarginal gyrus (SMG), and the middle temporal gyrus (MTG) showed an interesting pattern of FC. Echoes of the frontoparietal control networks and the default mode network (Fig. 4, ICN1, ICN2, ICN3, ICN6, ICN7) were detected within these regions, suggesting a potential location where their often-reported anticorrelation during attentional tasks (Fox et al., 2005) is established. Multisensory regions surrounding the TOPJ and MTG appear to communicate with sensorimotor, visual, and auditory networks (Fig. 4, ICN8, ICN9, ICN10). It is striking that whole-brain FC maps resembling the DMN could be found in $8 / 9$ peak regions, indicating that there is considerable overlap between the DMN and the multifunctional cortical hubs identified.

\section{Validation with ICNs from different databases}

We confirmed that our results were robust to different methods of defining the target ICNs. Figure 5 shows the results from correlating our FC maps with the whole-brain ICNs defined using our data, plus two independent target datasets based on rest and an aggregation of task data (Smith et al., 2009). All three datasets produced qualitatively similar maps with a similar spatial distribution of peak regions.

\section{Modulation of echoes during CRT task}

We went on to evaluate whether the subregions that we defined are functionally meaningful by studying how they are modulated during a simple cognitive task. A whole-brain searchlight analysis of the CRT data revealed a similar spatial distribution of regions to that shown in Figure 2 with the resting state data. However, the pattern of task modulation of neural signals within a given subregion differed substantially across different searchlights (Fig. 4).
The arrows superimposed on the similarity matrix in Figure 4 indicate which subregions showed a relative increase or decrease in activation for the CRT task compared with rest within the nine illustrative searchlights. Subregions with corresponding whole-brain FC maps consistent with visual and cognitive processing (higher level visual processing, ICN7; and a network including bilateral anterior insulae, parts of the salience network, ICN9) showed increased activity during the CRT. In contrast, subregions with whole-brain FC maps resembling the default mode (ICN1), left frontoparietal (ICN3), and primary visual regions (ICN10 and ICN11) showed relative decreases in activation. Different searchlights had different patterns of task modulation of subregional activity, with searchlights centered within DMN (i.e., the precuneus and PCC) displaying only patterns of relative deactivation (consistent with Leech et al., 2012). In contrast, other searchlights in regions typically associated with task-based activation increased with the CRT task (e.g., lateral occipital regions or TOPJ) and displayed increases in activation for some subregions (e.g., higher-order visual), whereas other subregions (resembling the DMN and primary visual regions) simultaneously showed decreases in activation.

Overlap of subregions is not necessary for whole-brain echoes The ICA-defined subregions within each searchlight displayed considerable spatial overlap with each other. To determine whether this overlap is necessary for the subregions to echo ICNs, the analysis was repeated within the PCC peak region, but with the subregions forced to be nonoverlapping. The 10 ICs from within the PCC were thresholded at an arbitrary threshold of $z>$ 3.5 and the overlapping voxels were removed. The nonoverlapping ICs were then entered into the dual-regression analysis as in Figure 1. Figure 7 show the resulting whole-brain FC maps for the overlapping and nonoverlapping subregion analyses, and it can be seen that the resulting maps are highly similar. This result suggests that the whole-brain echoes are the result of closely neighboring but nonoverlapping subregions.

\section{Discussion}

Our results suggest that heteromodal cortices contain subregions that echo the activity of multiple well characterized whole-brain functional networks. We have previously shown that this occurs within the PCC (Leech et al., 2012). Here, we show that this property is not unique to the PCC, but neither is it ubiquitous across the cortex. Instead, we see regional variability, with evidence of multiple echoes in ventromedial parietal cortex, right IPL, and bilateral regions of the TOPJ and dorsal ACC. These regions have been identified as cortical hubs based on structural and FC data (Hagmann et al., 2008; Tomasi and Volkow, 2010; Sepulcre et al., 2012). Here we propose how these regions mediate multiple functional signals: by replicating some of the temporal dynamics of the whole-brain ICNs within a local spatial organization of adjacent subregions. This suggests that these regions may be involved in integrating the information processed in the 


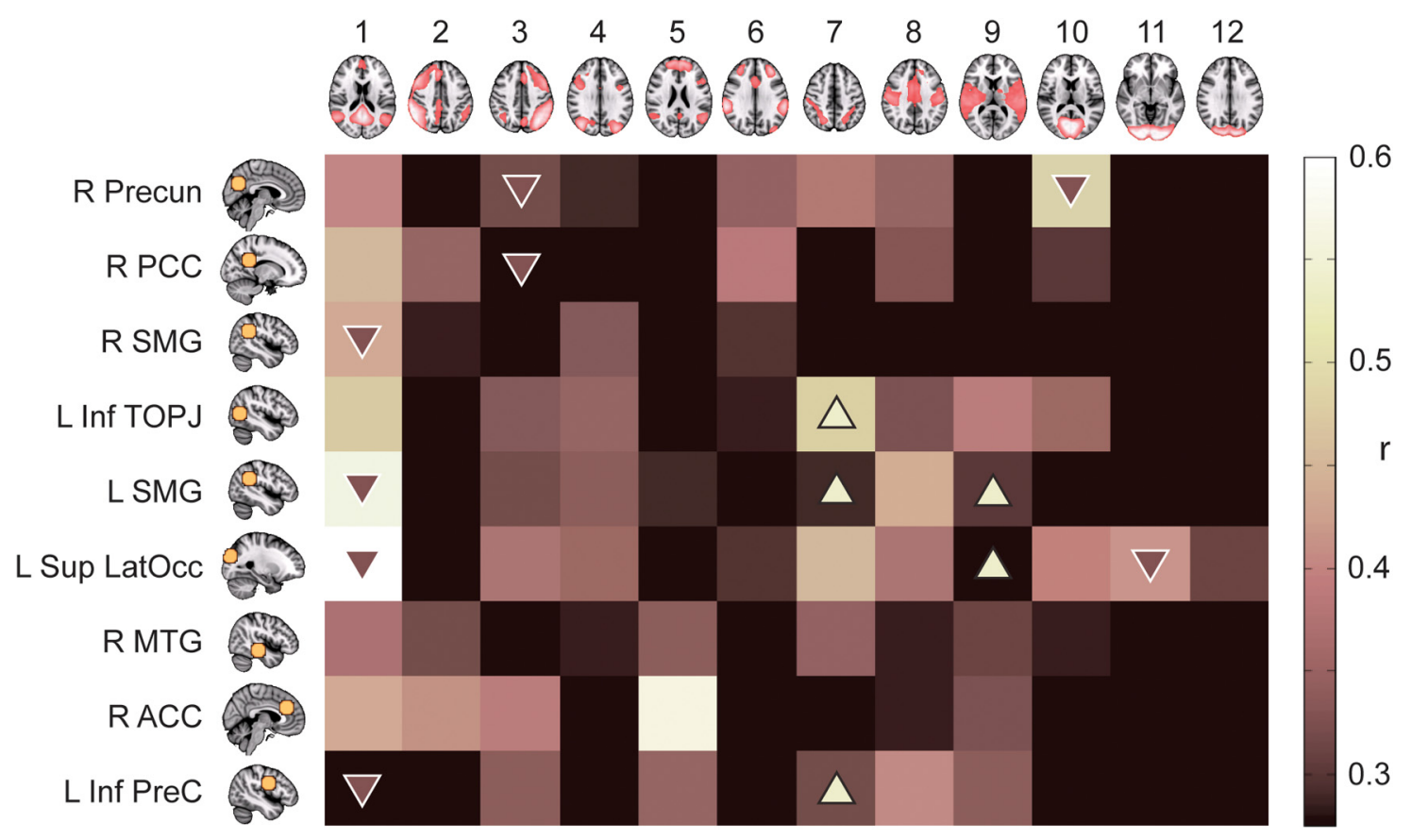

Figure 4. Correlation matrix between FC maps from the nine peak regions observed in Figure 2 (y-axis) and the resting state ICNs taken from Smith et al. (2009). ICN1 to ICN12 are shown on the $x$-axis. Each hub contained signals from distinct portfolios of the ICNs, suggesting they mediate the cross talk between different combinations of functional networks. The modulation of these multiple subregions during attentional engagement was assessed using a CRT task. Arrows indicate subregions where there was increased or decreased activity during attentive periods of the task compared with rest. Peak regions were (column from top to bottom, ordered by amount of spatial overlap with the default mode network); the right (R) precuneus (Precun), right PCC, right SMG, left (L) inferior TOPJ, left SMG, left superior lateral occipital cortex (Sup Lat0cc), right middle temporal gyrus (MTG), right ACC, and left inferior precentral gyrus (Inf PreC). ICN numbers (rows) are: 1, default mode; 2, right frontoparietal; 3, left frontoparietal; 4-6, cognitive control; 7, visual streams; 8, sensorimotor; 9 , salience plus auditory; 10, visual-1; 11, visual-2; and 12, visual-3 networks.

functionally specialized ICNs. Such integration is necessary for the hierarchical information processing that is essential to perception, cognition, and behavior, in keeping with Mesulam's suggested functional role of transmodal regions (Mesulam, 1998). Speculatively, some of these regions could be the location of the proposed global workspace (Tononi and Edelman, 1998; Dehaene and Naccache, 2001; Shanahan and Baars, 2005) necessary for high-level cognition and consciousness.

The set of whole-brain ICNs provides a coarse decomposition of the macroscopic neural activity measured with the BOLD signal from across the brain (Smith et al., 2009). We show that a subset of brain regions have a local topography (Jbabdi et al., 2013) that reflects the whole-brain ICNs (Fig. 6). One possible interpretation is that this subset of regions displays some degree of scale invariance (Expert et al., 2011).

The existence of echoes of multiple ICNs within this set of regions provides an explanation for recent work exploring $\mathrm{dy}$ namic interactions between brain regions (de Pasquale et al., 2012). There is considerable overlap between many of the regions we observed and areas showing low temporal coherence and high nonstationarity (Smith et al., 2012; de Pasquale et al., 2012) with other brain regions. The existence of multiple different signals in neighboring and presumably strongly interconnected regions could explain why such regions are highly temporally instable. At
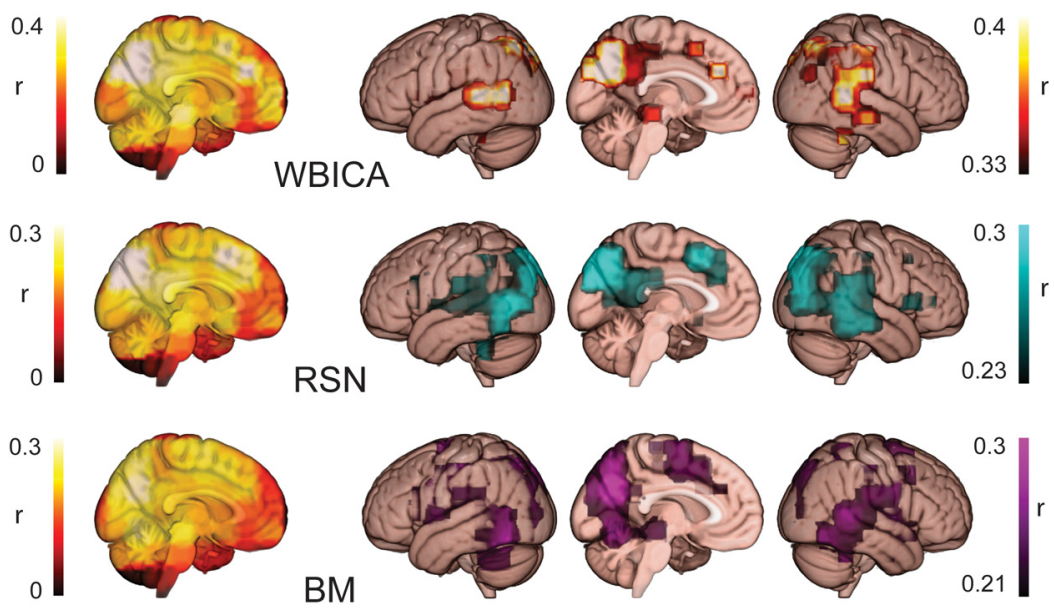

Figure 5. Correspondence between peak hub regions detected using ICNs obtained from different datasets. As with Figure 2, FC maps from each searchlight were correlated with ICNs from a WBICA of the same data and RSNs and grouped task-based data from BrainMap (BM) taken from Smith et al. (2009). Unthresholded (left) and thresholded (right) maps are included to illustrate the similarity in spatial distribution of the peak regions.

a larger spatial scale, the activation and FC of such regions may reflect the summation of these different signals. As a consequence, regions that contain more ICN signals may display a more variable aggregate time course and, over time, move in and out of coherence with a given ICN or cortical region.

Some of the regions echoing multiple ICNs overlap considerably with regions of the DMN, particularly the PCC and lateral inferior parietal cortices (Raichle and Snyder, 2007; Hagmann et al., 2008). Despite being one of the most prominent ICNs, the role of the DMN is still poorly understood. Our results suggest 


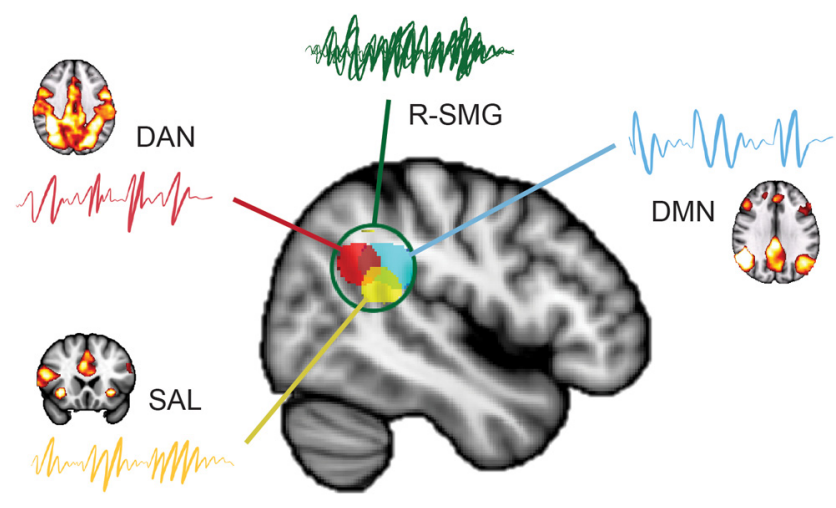

Figure 6. Echoes in the right SMG (R-SMG). Decomposition of the neural signal within the $\mathrm{R}$-SMG revealed multiple functional signals. These signals were strongest within small, separable but adjacent subregions of the R-SMG (red, yellow, and blue areas). Whole-brain FC (activation maps) revealed that the decomposed signals were echoes of intrinsic connectivity networks such as the default mode (DMN), salience (SAL), and dorsal attention (DAN) networks. This suggests that the R-SMG is uniquely placed to be able to coordinate and integrate the activity of various functional networks. Time series plots are illustrative of the overall activity being a combination of different signals.

the original sound that created it. However, although it is theoretically possible that the echo and the ICNs are functionally different (e.g., because they have different dynamics below the temporal resolution detectable with FMRI or because they both reflect a common undetectable third source), the echoes are likely to be parts of the whole-brain ICNs.

In our analysis, we identified several adjacent subregions echoing both frontoparietal control networks and the DMN, which are often anticorrelated during task (Fox et al., 2005). The presence of these subregions may explain why these regions are implicated in multiple cognitive states. The ACC is associated with reward anticipation, decision making, error processing, and motor control (Paus, 2001). The SMG and MTG are multisensory association areas that are activated during many tasks, including attention capture in multiple modalities (Driver and Spence, 1998; Shomstein and Yantis, 2006), top-down auditory attention (Langner et al., 2012; Braga et al., 2013), and language production/perception (Blank et al., 2002; Scott, 2012). The right MFG, part of the dorsolateral prefrontal cortex, displayed high heterogeneity in our analysis and has been implicated in many executive tasks, including working memory and attention (Cabeza and Nyberg, 2000; Corbetta et al., 2008). If the PCC properties discussed above are integral to all the heterogeneous regions identified, it is possible that, rather than being involved in multiple discrete functions (i.e., those attributed to the individual ICNs), these regions may serve primarily to maintain a critical balance between the activation of the different functional networks.

Although we found a network of regions showing echoes of multiple ICNs, we also show that different regions communicate with different subsets of the ICNs. This suggests that each region may have a unique role in integrating different types of information. For example, the right SMG showed restricted connectivity with the right lateralised frontoparietal

Figure 7. $F C$ maps from decomposition of the PCC searchlight into overlapping and nonoverlapping subregions. Similar FC maps were obtained using both types of subregions. Nonoverlapping subregions were obtained by thresholding the independent components from within the searchlight at an arbitrary threshold of $z>3.5$ and excluding voxels in which 2 or more components overlapped.

that the activity of core DMN regions is made up of overlapping neural signals from other ICNs. These signals, when summated over time, would produce the typical DMN appearance (Smith et al., 2012). This interpretation could explain the high metabolism normally detected in the DMN, because even at rest the intrinsic fluctuations of individual ICNs would each elicit trace activity within DMN hub regions. This would mean that at no point during rest or task would the whole DMN be fully "deactivated," because there are components of the signals that make up the DMN that are likely to be modulated even when the DMN shows overall decreased activity. Similarly, after task-based activation of a given ICN (e.g., of the right frontoparietal network), the balance of competing signals within DMN regions might be altered (Leech et al., 2012), which could explain why the DMN shows reduced overall activity during certain tasks. Our data suggest that central nodes of the DMN are strategically placed to modulate the cross talk between the ICNs, suggesting that the DMN may play a role in the dynamic integration of the information exchanged within functional networks.

We use the term "echo" because many of the subsignals we discuss are not detectable using conventional univariate seedbased FC analyses (Leech et al., 2012). They are less pronounced than in the central nodes of an ICN, just as an echo is quieter than network and dorsal and ventral visual streams (ICN4). This suggests the right SMG may be primarily involved in integrating visual sensory systems with executive/attentional systems. In contrast, the left SMG showed multiple sensory signals related to sensorimotor and auditory networks and the visual streams. This suggests that the left SMG is also involved in sensory integration, but may be less specialized for vision. Multisensory regions surrounding the TOPJ and MTG also appeared to be communicating with sensorimotor, visual, and auditory networks (ICN8, ICN9, and ICN10).

In the present study, we also analyzed data obtained during a simple, attentionally engaging task. By examining how the echoes were modulated by the task, we explored whether the subregions constitute a functionally meaningful subdivision and whether their modulation is consistent with existing theories about ICN recruitment. Consistent with the existing literature, we found increases in activation of echoes of ICNs relevant to cognitive tasks (i.e., higher-order visual networks and parts of the salience network) and deactivations predominantly of DMN echoes.

In addition to showing that the subregions are functionally meaningful, the task findings also suggest that: (1) across different searchlights, not all subregions echoing a given ICN were modulated by the task, which may indicate that, for example, the 
DMN echoes in certain parts of the brain are more responsive to task than in other regions; (2) several adjacent subregions can be simultaneously modulated by the task (sometimes in opposing directions), which hints that the local interactions between functionally distinct subregions may be critical for task performance; and (3) most of the subregions within each searchlight did not show any significant change in activation with the task, suggesting that attentional engagement only modulates activity in a subset of regions and that much of the activity in these regions is ongoing and unaffected. These findings show how the balance of activation within heteromodal regions is selectively altered during a cognitive engagement.

The phenomenon of different subregions within each searchlight echoing multiple whole-brain ICNs could be the result of at least two different types of local functional architecture. First, these regions could be constructed from single homogeneous circuits that interact with many different ICNs. Alternatively, these regions may be composed of multiple discrete but adjacent circuits, each communicating with different ICNs. By forcing the subregions to be nonoverlapping and showing that the resulting pattern of whole-brain FC was unchanged, we demonstrated that subregional overlap is not necessary. This suggests that the first hypothesis is unlikely to be the case and that heteromodal regions are better thought of as containing multiple discrete subregions. To better understand how the local functional architecture could support multiple ICNs, computational simulations modeled on biologically plausible brain network topologies (Deco et al., 2009) need to be devised.

In conclusion, we show here that many but not all regions of the human brain display considerable heterogeneity that results from communication with multiple functional networks simultaneously. We propose that the presence of echoes may explain why certain heteromodal regions (e.g., MFG, SMG) are repeatedly implicated in different functions and why the DMN may display the interesting metabolic properties (e.g., high resting metabolism, task-deactivation) that it does. Further work should determine whether these hub regions are involved in actively modulating the activity and coherence within the different ICNs and establish whether their role is truly integrative.

\section{References}

Beckmann CF, Jenkinson M, Smith SM (2003) General multilevel linear modeling for group analysis in FMRI. Neuroimage 20:1052-1063. CrossRef Medline

Beckmann CF, DeLuca M, Devlin JT, Smith SM (2005) Investigations into resting-state connectivity using independent component analysis. Philos Trans R Soc Lond B Biol Sci 360:1001-1013. CrossRef Medline

Beckmann CF, Mackay CE, Filippini N, Smith SM (2009) Group comparison of resting-state FMRI data using multi-subject ICA and dual regression. Paper presented at 15th Annual Meeting of the Organization for Human Brain Mapping, San Francisco, CA, June.

Blank SC, Scott SK, Murphy K, Warburton E, Wise RJ (2002) Speech production: Wernicke, Broca and beyond. Brain 125:1829-1838. CrossRef Medline

Bonnelle V, Leech R, Kinnunen KM, Ham TE, Beckmann CF, De Boissezon X, Greenwood RJ, Sharp DJ (2011) Default mode network connectivity predicts sustained attention deficits after traumatic brain injury. J Neurosci 31:13442-13451. CrossRef Medline

Braga RM, Wilson LR, Sharp DJ, Wise RJS, Leech R (2013) Separable networks for top-down attention to auditory non-spatial and visuospatial modalities. Neuroimage 74:77-86. CrossRef Medline

Bressler SL, Menon V (2010) Large-scale brain networks in cognition: emerging methods and principles. Trends Cogn Sci 14:277-290. CrossRef Medline

Cabeza R, Nyberg L (2000) Imaging cognition II: an empirical review of 275 PET and fMRI studies. J Cogn Neurosci 12:1-47. Medline
Corbetta M, Patel G, Shulman GL (2008) The reorienting system of the human brain: from environment to theory of mind. Neuron 58:306-324. CrossRef Medline

Damoiseaux JS, Greicius MD (2009) Greater than the sum of its parts: a review of studies combining structural connectivity and resting-state functional connectivity. Brain Struct Funct 213:525-533. CrossRef Medline

Deco G, Jirsa V, McIntosh AR, Sporns O, Kötter R (2009) Key role of coupling, delay, and noise in resting brain fluctuations. Proc Natl Acad Sci U S A 106:10302-10307. CrossRef Medline

Dehaene S, Naccache L (2001) Towards a cognitive neuroscience of consciousness: basic evidence and a workspace framework. Cognition 79:137. CrossRef Medline

de Pasquale F, Della Penna S, Snyder AZ, Marzetti L, Pizzella V, Romani GL, Corbetta M (2012) A cortical core for dynamic integration of functional networks in the resting human brain. Neuron 74:753-764. CrossRef Medline

Driver J, Spence C (1998) Crossmodal attention. Curr Opin Neurobiol 8:245-253. CrossRef Medline

Expert P, Lambiotte R, Chialvo DR, Christensen K, Jensen HJ, Sharp DJ, Turkheimer F (2011) Self-similar correlation function in brain restingstate functional magnetic resonance imaging. J R Soc Interface 8:472-479. CrossRef Medline

Fox MD, Snyder AZ, Vincent JL, Corbetta M, Van Essen DC, Raichle ME (2005) From The Cover: The human brain is intrinsically organized into dynamic, anticorrelated functional networks. Proc Natl Acad Sci U S A 102:9673-9678. CrossRef Medline

Fransson P (2005) Spontaneous low-frequency BOLD signal fluctuations: an fMRI investigation of the resting-state default mode of brain function hypothesis. Hum Brain Mapp 26:15-29. CrossRef Medline

Hagmann P, Cammoun L, Gigandet X, Meuli R, Honey CJ, Wedeen VJ, Sporns O (2008) Mapping the structural core of human cerebral cortex. PLoS Biol 6:e159. CrossRef Medline

Jbabdi S, Sotiropoulos SN, Behrens TE (2013) The topographic connectome. Curr Opin Neurobiol 23:207-215. CrossRef Medline

Langner R, Kellermann T, Eickhoff SB, Boers F, Chatterjee A, Willmes K, Sturm W (2012) Staying responsive to the world: modality-specific and -nonspecific contributions to speeded auditory, tactile, and visual stimulus detection. Hum Brain Mapp 33:398-418. CrossRef Medline

Leech R, Kamourieh S, Beckmann CF, Sharp DJ (2011) Fractionating the default mode network: distinct contributions of the ventral and dorsal posterior cingulate cortex to cognitive control. J Neurosci 31:3217-3224. CrossRef Medline

Leech R, Braga R, Sharp DJ (2012) Echoes of the brain within the posterior cingulate cortex. J Neurosci 32:215-222. CrossRef Medline

Margulies DS, Vincent JL, Kelly C, Lohmann G, Uddin LQ, Biswal BB, Villringer A, Castellanos FX, Milham MP, Petrides M (2009) Precuneus shares intrinsic functional architecture in humans and monkeys. Proc Natl Acad Sci U S A 106:20069-20074. CrossRef Medline

Mesulam M (2009) Defining neurocognitive networks in the BOLD new world of computed connectivity. Neuron 62:1-3. CrossRef Medline

Mesulam MM (1998) From sensation to cognition. Brain 121:1013-1052. CrossRef Medline

Murphy K, Birn RM, Handwerker DA, Jones TB, Bandettini PA (2009) The impact of global signal regression on resting state correlations: are anticorrelated networks introduced? Neuroimage 44:893-905. CrossRef Medline

Paus T (2001) Primate anterior cingulate cortex: where motor control, drive and cognition interface. Nat Rev Neurosci 2:417-424. CrossRef Medline

Raichle ME, Snyder AZ (2007) A default mode of brain function: a brief history of an evolving idea. Neuroimage 37:1083-1090; discussion 10971099. CrossRef Medline

Raichle ME, MacLeod AM, Snyder AZ, Powers WJ, Gusnard DA, Shulman GL (2001) Inaugural article: a default mode of brain function. Proc Natl Acad Sci U S A 98:676-682. CrossRef Medline

Scott SK (2012) The neurobiology of speech perception and productioncan functional imaging tell us anything we did not already know? J Commun Disord 45:419-425. CrossRef Medline

Sepulcre J, Sabuncu MR, Yeo TB, Liu H, Johnson KA (2012) Stepwise connectivity of the modal cortex reveals the multimodal organization of the human brain. J Neurosci 32:10649-10661. CrossRef Medline 
Shanahan M, Baars B (2005) Applying global workspace theory to the frame problem. Cognition 98:157-176. CrossRef Medline

Shomstein S, Yantis S (2006) Parietal cortex mediates voluntary control of spatial and nonspatial auditory attention. J Neurosci 26:435-439. CrossRef Medline

Simmonds AJ, Wise RJS, Collins C, Redzep O, Sharp DJ, Iverson P, Leech RL (2013) Parallel systems in the control of speech. Hum Brain Mapp.

Smith SM, Jenkinson M, Woolrich MW, Beckmann CF, Behrens TE, Johansen-Berg H, Bannister PR, De Luca M, Drobnjak I, Flitney DE, Niazy RK, Saunders J, Vickers J, Zhang Y, De Stefano N, Brady JM, Matthews PM (2004) Advances in functional and structural MR image analysis and implementation as FSL. Neuroimage 23:S208-S219. CrossRef Medline

Smith SM, Fox PT, Miller KL, Glahn DC, Fox PM, Mackay CE, Filippini N, Watkins KE, Toro R, Laird AR, Beckmann CF (2009) Correspondence of the brain's functional architecture during activation and rest. Proc Natl Acad Sci U S A 106:13040-13045. CrossRef Medline

Smith SM, Miller KL, Moeller S, Xu J, Auerbach EJ, Woolrich MW, Beckmann CF, Jenkinson M, Andersson J, Glasser MF, Van Essen DC, Feinberg DA, Yacoub ES, Ugurbil K (2012) Temporally-independent functional modes of spontaneous brain activity. Proc Natl Acad Sci U S A 109:3131-3136. CrossRef Medline

Sporns O, Honey CJ, Kötter R (2007) Identification and Classification of Hubs in Brain Networks. PLoS One 2:e1049. CrossRef Medline

Spreng RN, Stevens WD, Chamberlain JP, Gilmore AW, Schacter DL (2010) Default network activity, coupled with the frontoparietal control network, supports goal-directed cognition. Neuroimage 53:303-317. CrossRef Medline

Tomasi D, Volkow ND (2010) Functional connectivity density mapping. Proc Natl Acad Sci U S A 107:9885-9890. CrossRef Medline

Tononi G, Edelman GM (1998) Consciousness and complexity. Science 282:1846-1851. CrossRef Medline

Vogt BA, Vogt L, Laureys S (2006) Cytology and functionally correlated circuits of human posterior cingulate areas. Neuroimage 29: 452-466. CrossRef Medline

Zuo XN, Kelly C, Adelstein JS, Klein DF, Castellanos FX, Milham MP (2010) Reliable intrinsic connectivity networks: test-retest evaluation using ICA and dual regression approach. Neuroimage 49:2163-2177. CrossRef Medline 